
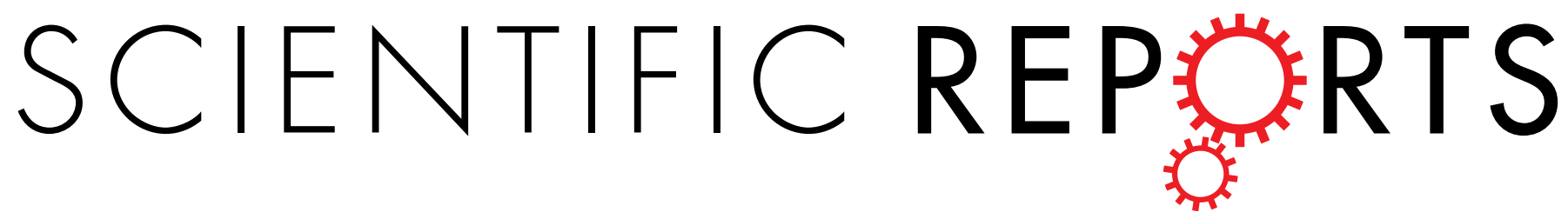

\title{
OPEN Nature of the Insulating Ground State of the Two-Dimensional Sn Atom Lattice on SiC(0001)
}

Received: 27 April 2016

Accepted: 04 July 2016

Published: 28 July 2016

\section{Seho $\mathrm{Yi}^{1}$, Hunpyo Lee ${ }^{2}$, Jin-Ho Choi ${ }^{1,3}$ \& Jun-Hyung Cho ${ }^{1}$}

Semiconductor surfaces with narrow surface bands provide unique playgrounds to search for Mottinsulating state. Recently, a combined experimental and theoretical study of the two-dimensional (2D) $\mathrm{Sn}$ atom lattice on a wide-gap $\mathrm{SiC}(0001)$ substrate proposed a Mott-type insulator driven by strong on-site Coulomb repulsion $U$ within a single-band Hubbard model. However, our systematic densityfunctional theory (DFT) study with local, semilocal, and hybrid exchange-correlation functionals shows that the Sn dangling-bond state largely hybridizes with the substrate Si $3 p$ and C $2 p$ states to split into three surface bands due to the crystal field. Such a hybridization gives rise to the stabilization of the antiferromagnetic order via superexchange interactions. The band gap and the density of states predicted by the hybrid DFT calculation agree well with photoemission data. Our findings not only suggest that the $\mathrm{Sn} / \mathrm{SiC}(0001)$ system can be represented as a Slater-type insulator driven by long-range magnetism, but also have an implication that taking into account long-range interactions beyond the on-site interaction would be of importance for properly describing the insulating nature of $\mathrm{Sn} / \mathrm{SiC}(0001)$.

Search for Mott-insulating state driven by short-range electron correlations has long been one of the most challenging issues in condensed matter physics ${ }^{1,2}$. Since the electrons in two-dimensional (2D) atom lattices can experience strong on-site Coulomb repulsion $U$ due to their reduced screening, metal overlayers on semiconductor substrates have attracted much attention for the realization of a Mott-Hubbard insulator ${ }^{3-9}$, where $U$ splits a half-filled band into lower and upper Hubbard bands. For example, the 1/3-monolayer adsorption of Sn atoms on the $\mathrm{Si}(111)$ or $\mathrm{Ge}(111)$ surface produces the $\sqrt{3} \times \sqrt{3}$ reconstruction in which all the dangling bonds (DBs) of underlying Si or Ge surface atoms are saturated to leave a single DB on each Sn atom ${ }^{5-14}$. Such Sn-overlayer systems with a half-filled band have been considered as an ideal playground for investigating $2 \mathrm{D}$ correlated physics on the $\sqrt{3} \times \sqrt{3}$ triangular lattice ${ }^{5-9}$. However, the nature of the insulating ground state in $\mathrm{Sn} / \mathrm{Si}(111)$ or Sn/ $\mathrm{Ge}(111)$ has become a controversial issue whether the gap formation is driven by strong Coulomb interactions (Mott-type insulator) $^{5-9}$ or by long-range magnetic order (Slater-type insulator) ${ }^{13,14}$.

To realize a significantly reduced adatom-substrate hybridization as well as a strongly suppressed screening, Glass et al..$^{15}$ fabricated the $\sqrt{3} \times \sqrt{3}$ phase of Sn overlayer on a wide-gap SiC(0001) substrate (see Fig. 1a,b). In their photoemission experiment on the $\mathrm{Sn} / \mathrm{SiC}(0001)$ surface system, Glass et al. observed a large energy gap of $\sim 2 \mathrm{eV}$. To account for the origin of such an insulating phase, Glass et al. performed the combined density -functional theory and dynamical mean-field theory (DFT + DMFT) calculations for a single-band Hubbard model that includes only the on-site Coulomb repulsion, and reproduced the experimentally observed insulating gap. Meanwhile, their spin-polarized DFT calculation ${ }^{15}$ with the local density approximation (LDA) predicted a small energy gap of $\sim 0.1 \mathrm{eV}$ for the collinear antiferromagnetic (AFM) ordering. Based on these results, Glass et al. interpreted the $\mathrm{Sn} / \mathrm{SiC}(0001)$ surface system as a pronounced Mott-type insulator. However, the theoretical analysis of Glass et al..${ }^{15}$ leading to the Mott-insulating scenario raises the following questions: (i) Is the single-band Hubbard model employed in the previous DFT + DMFT calculation ${ }^{15}$ suitable for describing the insulating nature of the $\mathrm{Sn} / \mathrm{SiC}(0001)$ system? and (ii) Does the LDA accurately predict the insulating gap formed by the AFM order?

${ }^{1}$ Department of Physics and Research Institute for National Sciences, Hanyang University, 17 Haengdang-Dong, Seongdong-Ku, Seoul 133-791, Korea. ${ }^{2}$ School of General Studies, Kangwon National University, 346 Jungang-ro, Samcheok-si, Kangwon-do, Korea. ${ }^{3}$ Research Institute of Mechanical Technology, Pusan National University, 30 Jangjeon-dong, Geumjeong-gu, Pusan 609-735, Korea. Correspondence and requests for materials should be addressed to J.-H.C. (email: chojh@hanyang.ac.kr) 
(a)

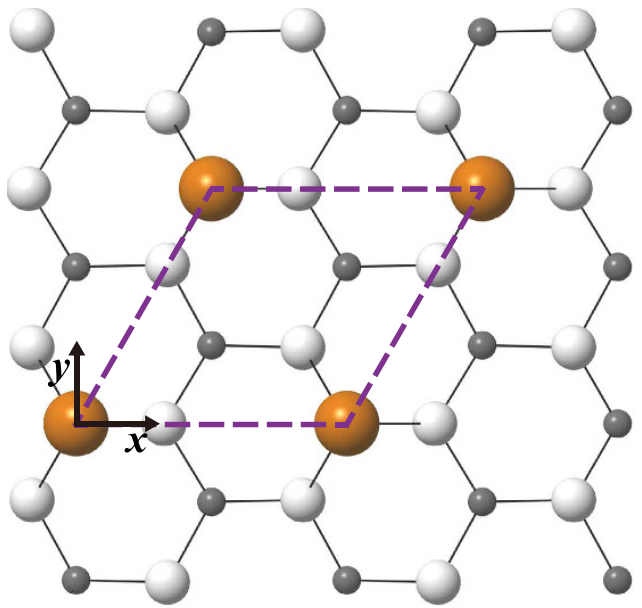

(b)

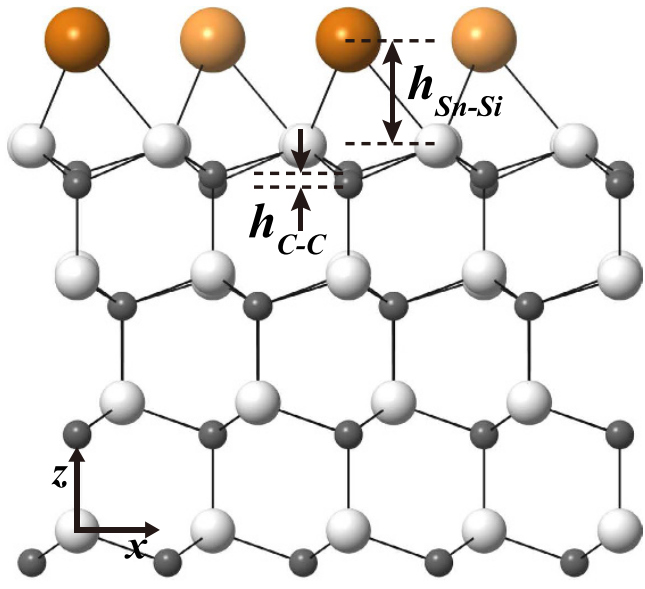

Figure 1. Atomic geometry of $\mathrm{Sn} / \mathrm{SiC}(\mathbf{0 0 0 1})$. Top (a) and side (b) views of the structure of $\mathrm{Sn} / \mathrm{SiC}(0001)$. The dashed line indicates the $\sqrt{3} \times \sqrt{3}$ unit cell. The $\mathbf{x}, \mathbf{y}$, and $\mathbf{z}$ axes point along the [1000], [01 10$]$, and [0001] directions, respectively. The large, medium, and small circles represent $\mathrm{Sn}, \mathrm{Si}$, and $\mathrm{C}$ atoms, respectively. For distinction, Sn atoms on the different $y$ positions are drawn with dark and bright circles in the side view.

We investigate the nature of the insulating ground state of $\mathrm{Sn} / \mathrm{SiC}(0001)$ by using the systematic DFT calculations with the LDA, semilocal generalized gradient approximation (GGA), and hybrid exchange-correlation functionals as well as the LDA + DMFT calculation. All of the DFT calculations predict the AFM ground state, but the calculated band gap largely depends on the employed exchange-correlation functionals. Specifically, the hybrid DFT results for the band gap and the density of states (DOS) agree well with photoemission data. It is revealed that the $S n 5 p_{x}, 5 p_{y}$, and $5 p_{z}$ orbitals largely hybridize with the substrate $\mathrm{Si} 3 p_{z}$ and $\mathrm{C} 2 p_{z}$ orbitals, leading to three surface bands due to the crystal-field splitting. Such an unexpectedly large hybridization between the Sn DB state and the substrate states not only facilitates the superexchange interactions between neighboring Sn atoms to stabilize the AFM order, but also implies that long-range interactions beyond the on-site interaction should be taken into account for properly describing the insulating nature of $\mathrm{Sn} / \mathrm{SiC}(0001)$. The present results suggest that the $\mathrm{Sn} / \mathrm{SiC}(0001)$ surface system can be more represented as a Slater-type insulator via long-range magnetism rather than the previously ${ }^{15}$ proposed Mott-type insulator via strong on-site Coulomb repulsion.

\section{Results}

We begin to optimize the atomic structure of the nonmagnetic $(\mathrm{NM}) \sqrt{3} \times \sqrt{3}$ structure using the LDA, GGA, and hybrid DFT calculations. The optimized NM structure obtained using LDA is displayed in Fig. 1 . We find that within the LDA scheme the height difference between the $\mathrm{Sn}$ atom and its bonding $\mathrm{Si}$ atoms $\left(h_{\mathrm{Sn}-\mathrm{Si}}\right)$ is $2.09 \AA$ and that between the first C-layer atoms $\left(h_{\mathrm{C}-\mathrm{C}}\right)$ is $0.19 \AA$, in good agreement with those $\left(h_{\mathrm{Sn}-\mathrm{Si}}=2.03 \AA\right.$ and $h_{\mathrm{C}-\mathrm{C}}=0.21 \AA$ ) of a previous LDA calculation ${ }^{15}$. The values of $h_{\mathrm{Sn}-\mathrm{Si}}$ and $h_{\mathrm{C}-\mathrm{C}}$ slightly change by less than $0.05 \AA$, depending on the employed exchange-correlation functionals. Figure $2 \mathrm{a}$, b show the LDA band structure and partial density of states (PDOS) projected onto the Sn $5 p$ and substrate Si $3 p$ and C $2 p$ orbitals, respectively. Interestingly, we find that $S n \mathrm{DB}$ electrons form three surface bands designated as $S_{1}, S_{2}$, and $S_{3}$ (see Fig. 2a). The bands projected onto the Sn $5 p$, Si $3 p$, and C $2 p$ orbitals show that the $S_{1}$ band is composed of $\sim 50 \%$ Sn $5 p_{z}$ orbital, $\sim 25 \% \mathrm{Si} 3 p_{z}$ orbital, and $\sim 25 \% \mathrm{C} 2 p_{z}$ orbital, while the $S_{2}$ and $S_{3}$ bands are composed of $\sim 40 \%$ Sn $5 p_{x}$ and $5 p_{y}$ orbitals, $\sim 30 \%$ Si $3 p$ orbitals, and $\sim 30 \%$ C $2 p$ orbitals (see Fig. $2 b$ ). Since the Sn $5 p_{z}$ orbital has more spatial overlap with the topmost substrate $\mathrm{Si} 3 p_{z}$ or C $2 p_{z}$ orbital compared to that between the Sn $5 p_{x}\left(\right.$ or $5 p_{y}$ ) and the topmost substrate $\mathrm{Si} 3 p$ or C $2 p$ orbitals, the former overlap is likely to give rise to a larger electrostatic repulsion than the latter one. Such a crystal-field splitting causes the higher energy of the $S_{1}$ state relative to the almost degenerate $S_{2}$ and $S_{3}$ states. It is noted that the large hybridization between the Sn DB state and the substrate Si and C states is well reflected by the conspicuously mixed charge character (see Fig. 2c), which exhibits some localization of DB electrons around $\mathrm{Sn}$ atom as well as a large delocalization of $\mathrm{Si} 3 p_{z}$ or $\mathrm{C} 2 p_{z}$ electrons up to the sixth deeper substrate layer.

As shown in Fig. 2a, the $S_{1}$ state crosses the Fermi level $E_{F}$, producing a half-filled band. Despite its delocalized charge character as mentioned above, the $S_{1}$ state has a small band width of $0.31,0.33$, and $0.55 \mathrm{eV}$, obtained using the LDA, GGA, and hybrid DFT calculations, respectively. This flat-band-like feature is likely to be attributed to a large separation of $\sim 5.3 \AA$ between $S n$ atoms within the $\sqrt{3} \times \sqrt{3}$ unit cell. Because of such a narrow band width of the $S_{1}$ state, the electronic instabilities such as a charge or spin density wave (CDW/SDW) may be expected. For the CDW instability, we find that the $3 \times 3$ structure containing three $\mathrm{Sn}$ atoms (i.e., $\mathrm{U}_{1}, \mathrm{U}_{2}$, and $\mathrm{D}$ atoms in Fig. S1 of the Supplementary Information) of different heights is more stable than the NM $\sqrt{3} \times \sqrt{3}$ structure by 13.4, 19.0, and $182.8 \mathrm{meV}$ per $\sqrt{3} \times \sqrt{3}$ unit cell for LDA, GGA, and hybrid DFT, respectively (see Table 1). 
(a)

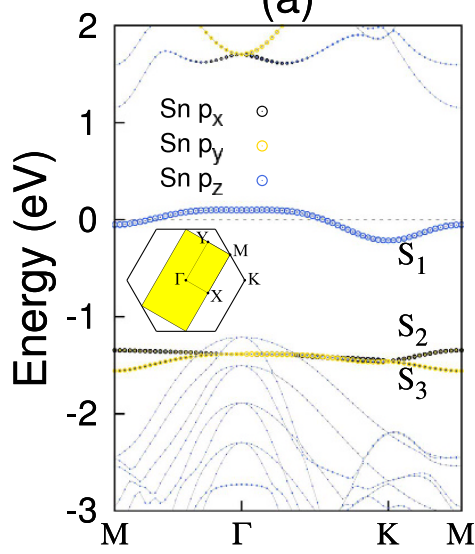

(b)

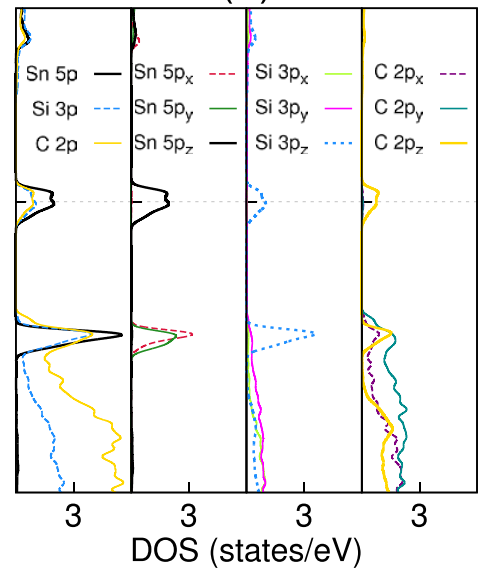

(c)

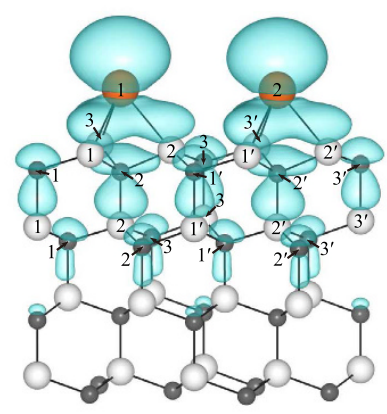

Figure 2. Calculated band structure, partial DOS and eigenstate density of NM $\sqrt{3} \times \sqrt{3}$ structure. (a) The Sn $p$-orbitals projected band structure, (b) the partial DOS projected onto the Sn $5 p$, Si $3 p$, and C $2 p$ orbital of the NM $\sqrt{3} \times \sqrt{3}$ structure and (c) the charge character of the $S_{1}$ state at the $\Gamma$ point, obtained using the LDA functional. The Brillouin zones of the NM $\sqrt{3} \times \sqrt{3}$ structures are drawn in the inset of (a). The energy zero represents the Fermi level. The charge character is displayed with an isosurface of $0.002 e / \AA^{3}$.

\begin{tabular}{|l|c|c|c|}
\hline & CDW & FM & AFM \\
\hline LDA & -13.4 & -16.1 & -28.7 \\
\hline GGA & -19.0 & -61.8 & -69.3 \\
\hline hybrid DFT & -182.8 & -410.8 & -446.5 \\
\hline
\end{tabular}

Table 1. Total energies of the CDW, FM and AFM structures relative to the NM structure. The total energies (in meV per $\sqrt{3} \times \sqrt{3}$ unit cell) calculated using LDA, GGA and hybrid DFT.

Since such a buckled NM $3 \times 3$ structure accompanies a charge transfer from the $\mathrm{D}$ to the $\mathrm{U}_{1}$ (or $\left.\mathrm{U}_{2}\right)$ atoms, it is most likely to reduce Coulomb repulsions between Sn DB electrons compared to the NM $\sqrt{3} \times \sqrt{3}$ structure. We note that the calculated band structure of the NM $3 \times 3$ structure exhibits the presence of occupied surface states at $E_{F}$ (see Fig. S1 of the Supplementary Information), indicating a metallic feature. To find the possibility of SDW, we perform the spin-polarized LDA, GGA, and hybrid DFT calculations for the ferromagnetic (FM) $\sqrt{3} \times \sqrt{3}$ and AFM $2 \sqrt{3} \times \sqrt{3}$ structures, which were considered in the previous LDA calculation ${ }^{15}$. We find that all of the employed exchange-correlation functionals energetically favor the FM and AFM structures over the $\mathrm{NM} \sqrt{3} \times \sqrt{3}$ and $3 \times 3$ structures (see Table 1 ). Here, the AFM structure is more stable than the FM structure, consistent with the previous LDA calculation ${ }^{15}$. It is noted that the stabilities of the two magnetic structures relative to the NM $\sqrt{3} \times \sqrt{3}$ structure increase in the order of LDA $<$ GGA $<$ hybrid DFT calculations (see Table 1). In the optimized AFM structure, two Sn atoms within the $2 \sqrt{3} \times \sqrt{3}$ unit cell are at the same height, indicating a $\sqrt{3} \times \sqrt{3}$ structural symmetry as observed by low-energy electron diffraction and scanning tunneling microscopy ${ }^{15}$.

Figure $3 \mathrm{a}-\mathrm{c}$ show the LDA, GGA, and hybrid-DFT band structures of the AFM structure, which give the band gap $E_{g}$ of $0.12,0.30$, and $1.97 \mathrm{eV}$, respectively. The band gap obtained using hybrid DFT is found to be closer to that $(\sim 2 \mathrm{eV})$ measured by photoemission spectroscopy ${ }^{15}$. As shown in Fig. 3c, the DOS obtained using hybrid DFT exhibits the three peaks located at $-1.09,-1.83$, and $-2.17 \mathrm{eV}$ below $E_{F}$, which are associated with the $S_{1}, S_{2}$, and $S_{3}$ states, respectively. On the other hand, photoemission spectra ${ }^{15}$ showed the presence of two peaks at -1.0 and $-2.4 \mathrm{eV}$, which were interpreted to originate from the Sn DB state and the SiC bulk states, respectively. Based on the present DOS results, we however interpret the upper and lower photoemission peaks in terms of the $S_{1}$ and $S_{2}\left(\right.$ or $S_{3}$ ) surface states, respectively.

To understand the underlying mechanism for the gap opening of the AFM spin ordering, we plot in Fig. 3d the spin-polarized local DOS projected onto the two Sn atoms at A and B sites, together with their spin characters. It is seen that the occupied (unoccupied) spin-up and spin-down $S_{1}$ states are localized at the A(B) and $\mathrm{B}(\mathrm{A})$ sites, respectively. Here, the hybridization between the occupied spin-up (spin-down) state at the $\mathrm{A}(\mathrm{B})$ site and the unoccupied spin-up (spin-down) state at the $\mathrm{B}(\mathrm{A})$ site gives rise to a gap opening ${ }^{16}$. Such superexchange interaction ${ }^{17,18}$ between the occupied and unoccupied electronic states can be facilitated due to a large hybridization of the Sn $5 p_{z}$ orbitals with the Si $3 p_{z}$ and C $2 p_{z}$ orbitals (see Fig. 2b). This superexchange interaction is well represented by a large spin delocalization (see Fig. $3 \mathrm{~d}$ ) with the spin moments of $\pm 0.33, \pm 0.12$, and $\pm 0.10 \mu_{B}$ for $\mathrm{Sn}, \mathrm{Si}$ (outermost-layer) and C (outermost-layer) atoms, respectively (see the hybrid DFT results in Table 2). We note that the calculated spin moments of $\mathrm{Sn}, \mathrm{Si}$, and $\mathrm{C}$ atoms increase in the order of $\mathrm{LDA}<\mathrm{GGA}<$ hybrid DFT 
(a)

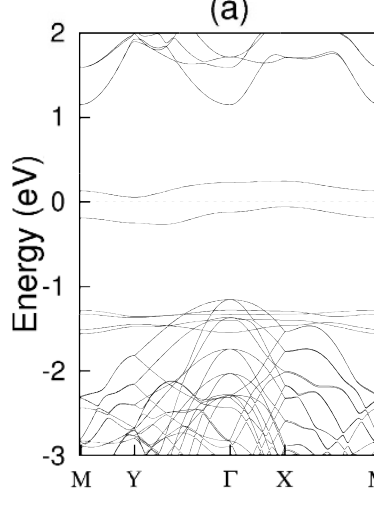

(b)

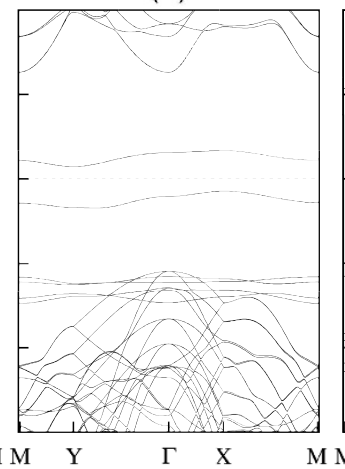

(c)

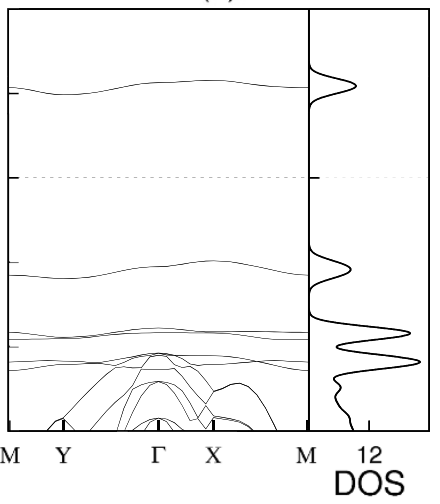

(d)

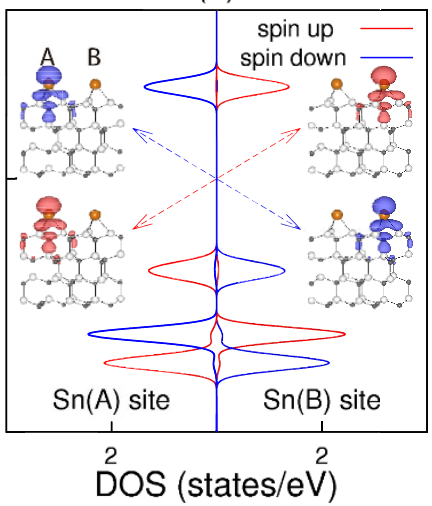

Figure 3. Band structure of the AFM strucure. The band dispersions obtained using (a) LDA, (b) GGA, and (c) hybrid DFT are plotted along the symmetry lines in the surface Brillouin zone of the unit cell (see the inset in Fig. 2a). In (c), the DOS is also given. The spin-polarized local DOS projected onto the two Sn atoms at the A and B sites within the AFM structure, obtained using hybrid DFT, are given in (d). Here, the charge characters of the spin-up (spin-down) states for the highest occupied and the lowest unoccupied bands are taken at the $\Gamma$ point with an isosurface of $0.002(-0.002) e / \AA^{3}$.

\begin{tabular}{|l|c|c|c|c|c|c|}
\hline Sn atoms & \multicolumn{3}{|c|}{$\mathbf{S n}_{\mathbf{1}} \mathbf{0 . 3 3 4}(\mathbf{0 . 2 8 4 , 0 . 2 9 1 )}$} & \multicolumn{2}{c|}{$\mathrm{Sn}_{\mathbf{2}}-\mathbf{0 . 3 3 4}(-\mathbf{0 . 2 8 4},-\mathbf{0 . 2 9 1})$} \\
\hline 1st layer & $\begin{array}{c}\mathrm{Si}_{1} 0.039 \\
(0.029,0.031)\end{array}$ & $\begin{array}{c}\mathrm{Si}_{2} 0.039 \\
(0.029,0.031)\end{array}$ & $\begin{array}{c}\mathrm{Si}_{3} 0.039 \\
(0.029,0.031)\end{array}$ & $\begin{array}{c}\mathrm{Si}_{1^{\prime}}-0.039 \\
(-0.029,-0.031)\end{array}$ & $\begin{array}{c}\mathrm{Si}_{2^{\prime}}-0.039 \\
(-0.029,-0.031)\end{array}$ & $\begin{array}{c}\mathrm{Si}_{3^{\prime}}-0.039 \\
(-0.029,-0.031)\end{array}$ \\
\hline 2nd layer & $\begin{array}{c}\mathrm{C}_{1} 0.004 \\
(0.004,0.004)\end{array}$ & $\begin{array}{c}\mathrm{C}_{2} 0.091 \\
(0.081,0.086)\end{array}$ & $\begin{array}{c}\mathrm{C}_{3} 0.004 \\
(0.004,0.004)\end{array}$ & $\begin{array}{c}\mathrm{C}_{1^{\prime}}-0.004 \\
(-0.004,-0.004)\end{array}$ & $\begin{array}{c}\mathrm{C}_{2^{\prime}}-0.091 \\
(-0.081,-0.086)\end{array}$ & $\begin{array}{c}\mathrm{C}_{3^{\prime}}-0.004 \\
(-0.004,-0.004)\end{array}$ \\
\hline 3rd layer & $\begin{array}{c}\mathrm{Si}_{1} 0.001 \\
(0.001,0.001)\end{array}$ & $\begin{array}{c}\mathrm{Si}_{2} 0.037 \\
(0.033,0.036)\end{array}$ & $\begin{array}{c}\mathrm{Si}_{3} 0.001 \\
(0.001,0.001)\end{array}$ & $\begin{array}{c}\mathrm{Si}_{1^{\prime}}-0.001 \\
(-0.001,-0.001)\end{array}$ & $\begin{array}{c}\mathrm{Si}_{2^{\prime}}-0.037 \\
(-0.033,-0.036)\end{array}$ & $\begin{array}{c}\mathrm{Si}_{3^{\prime}}-0.001 \\
(-0.001,-0.001)\end{array}$ \\
\hline 4th layer & $\begin{array}{c}\mathrm{C}_{1} 0.003 \\
(0.002,0.003)\end{array}$ & $\begin{array}{c}\mathrm{C}_{2} 0.003 \\
(0.002,0.003)\end{array}$ & $\begin{array}{c}\mathrm{C}_{3} 0.003 \\
(0.002,0.003)\end{array}$ & $\begin{array}{c}\mathrm{C}_{1^{\prime}}-0.003 \\
(-0.002,-0.003)\end{array}$ & $\begin{array}{c}\mathrm{C}_{2^{\prime}}-0.003 \\
(-0.002,-0.003)\end{array}$ & $\begin{array}{c}\mathrm{C}_{3^{\prime}}-0.003 \\
(-0.002,-0.003)\end{array}$ \\
\hline
\end{tabular}

Table 2. Spin magnetic moments of AFM structure. Calculated spin magnetic moments (in $\mu_{B}$ ) within the PAW spheres centered at $\mathrm{Sn}, \mathrm{Si}$, and $\mathrm{C}$ atoms, obtained using the HSE calculation. The PAW sphere radii were chosen as $1.566,1.312$, and $0.863 \AA$ for $\mathrm{Sn}, \mathrm{Si}$, and $\mathrm{C}$ atoms, respectively. The numbering of each atom is seen in the Fig. $2 \mathrm{c}$ above. The results obtained by using the LDA and GGA calculations are also given in parentheses as $\left(m_{L D A}, m_{G G A}\right)$.

calculations (see Table 2), which is the same as the order of the relative energies shown in Table 1. On the basis of our DFT calculations, we can say that the magnetically driven insulating state of $\mathrm{Sn} / \mathrm{SiC}(0001)$ with a large spin delocalization can be characterized as a Slater-type insulator.

The existence of the long-range AFM order due to the sizable hybridization between the Sn DB state and the substrate states raises questions about the reliability of the previous LDA + DMFT study ${ }^{15}$ in which a single-band Hubbard model including only the on-site Coulomb interaction was employed. Here, the single band representing the DB state dominantly localized at Sn atoms invokes strong on-site Coulomb repulsion with suppressed electron hoping, driving the gap formation. Despite the fact that such a model Hamiltonian does not incorporate long-range interactions due to the largely hybridized $S_{1}$ state, we solve it within the LDA + DMFT scheme. Figure 4a shows the calculated DOS for the AFM and paramagnetic phases obtained at $T=100$ and $300 \mathrm{~K}$, respectively. The observed insulating gap of $\sim 2 \mathrm{eV}$ is found to be well reproduced with $U=1.8 \mathrm{eV}$, similar to the previous ${ }^{15}$ LDA + DMFT calculation. As shown in Fig. 4b, the paramagnetic phase is transformed into the AFM phase below $T_{N} \approx 100 \mathrm{~K}$. Note that such a phase transition little changes the insulating gap (see Fig. $4 \mathrm{a}$ ). Therefore, the LDA + DMFT results indicate that the gap formation is not driven by the AFM order but attributed to the on-site interaction, representing a Mott-type insulator. Accordingly, the spin magnetic moment obtained using $\mathrm{LDA}+\mathrm{DMFT}$ is $1 \mu_{B}$ for Sn atom (see Fig. 4b). Such a localized magnetic moment inherent in the Mott phase drastically contrasts with the large spin delocalization over $\mathrm{Sn}$ atoms and Si substrate atoms obtained using the hybrid DFT calculation (see Fig. 3d and Table 2). Future experiments are anticipated to resolve such different features of spin magnetic moment between the Mott-type and Slater-type insulators by measuring the surface magnetic moments at $\mathrm{Sn} / \mathrm{SiC}(0001)$.

\section{Discussion}

The charge character of the $S_{1}$ state exhibits a large delocalization up to the third deeper Si and C substrate layers, which in turn gives some lateral overlap between neighboring Sn atoms (see the inset of Fig. 2a). Such an extension of the half-filled surface state calls for the importance of long-range interactions which were not considered in the previous ${ }^{15}$ and present LDA + DMFT calculations. It is noted that long-range interactions include inter-site Coulomb interactions, charge ordering, and next-nearest neighbor hopping. Indeed, a recent fully self-consistent 
(a)

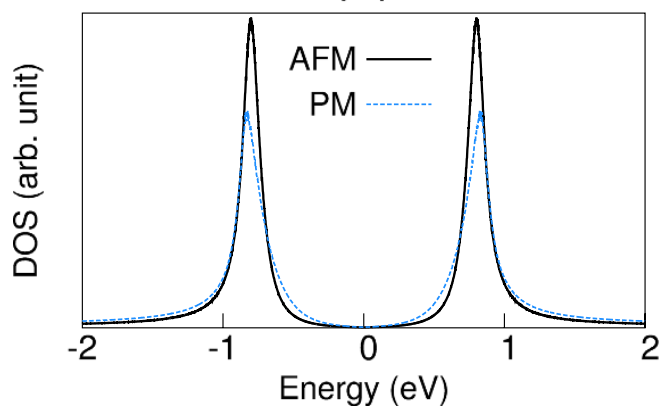

(b)

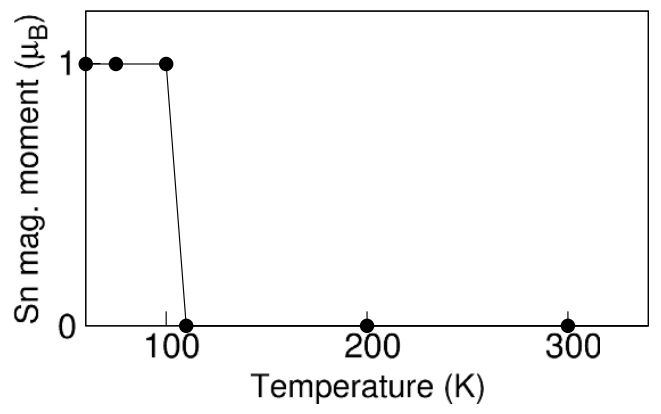

Figure 4. LDA + DMFT calculation with $U=1.8 \mathrm{eV}$. (a) Density of states of the AFM and paramagnetic (PM) phases obtained at 100 and $300 \mathrm{~K}$, respectively. The calculated spin magnetic moment of $\mathrm{Sn}$ atom is plotted as a function of temperature in $(\mathbf{b})$.

$\mathrm{GW}+\mathrm{DMFT}$ study ${ }^{19}$ for the analogous $\mathrm{X} / \mathrm{Si}(111)$ systems (with $\mathrm{X}=\mathrm{C}, \mathrm{Si}, \mathrm{Sn}$, and $\mathrm{Pb}$ ) reported that taking into account inter-site Coulomb interactions is mandatory because of their comparable magnitude with that of the on-site Coulomb interaction. Such an inclusion of inter-site interactions within the extended Hubbard model changes the ground-state character of the $\mathrm{X} / \mathrm{Si}(111)$ systems ${ }^{19}$ : i.e., without inter-site interactions, all the $\mathrm{X} / \mathrm{Si}(111)$ systems are in the Mott phase, but, as they are added, $\mathrm{Sn} / \mathrm{Si}(111)$ and $\mathrm{Pb} / \mathrm{Si}(111)$ become closer to a metallic phase. Compared to the $\mathrm{Sn} / \mathrm{Si}(111)$ system, $\mathrm{Sn} / \mathrm{SiC}(0001)$ has the $\sim 20 \%$ smaller nearest-neighbor distance of Sn atoms as well as the relatively lower dielectric screening of the SiC substrate, thereby leading to an increase in the inter-site interactions. It is thus expected that the nonlocal interaction effects in $\mathrm{Sn} / \mathrm{SiC}(0001)$ might significantly influence the stability of the Mott phase obtained by using only the on-site interaction. For more accurate simulation of the present system, the extended Hubbard model including long-range interaction terms will be demanded in future theoretical work. There still remains an interesting challenge of how to equally consider all of the on-site interaction, long-range interactions, and magnetic response in the $\mathrm{Sn} / \mathrm{SiC}(0001)$ system.

To conclude, we have presented two different pictures for the insulating nature of the Sn overlayer on a wide-gap $\mathrm{SiC}(0001)$ substrate using the LDA, GGA, and hybrid DFT calculations and the LDA + DMFT calculation. The DFT calculations drew the Slater-type picture with a long-range AFM order, while the LDA + DMFT calculation supported the Mott-type picture driven by strong on-site Coulomb repulsion. Unexpectedly, the Sn DB state was found to largely hybridize with the substrate Si and C states, thereby facilitating the stabilization of the AFM spin ordering via superexchange interactions. This intriguing electronic structure of the present system raises an important issue of how long-range interactions beyond the on-site interaction should be taken into account to diminish the Mott phase. Our findings will not only caution against the realization of the Mott-insulating phase in metal overlayers on semiconductor substrates, but also stimulate further experimental studies for the exploration of the magnetic phases of $\mathrm{Sn} / \mathrm{SiC}(0001)$.

\section{Methods}

Density Functional Theory calculations. We have performed the LDA, GGA, and hybrid DFT calculations using the Vienna ab initio simulation package (VASP) with the projector augmented wave method ${ }^{20,21}$. For the exchange-correlation energy, we employed the LDA functional of Ceperley-Alder (CA ${ }^{22}$, the GGA functional of Perdew-Burke-Ernzerhof $(\mathrm{PBE})^{23}$, and the hybrid functional of Heyd-Scuseria-Ernzerhof $(\mathrm{HSE})^{24,25}$. Since the HSE functional with a mixing factor of $\alpha=0.5$ controlling the amount of exact Fock exchange energy predicts well the observed insulating gap of $\sim 2 \mathrm{eV}$, we used this optimal value $\alpha_{\text {opt }}$ for the hybrid DFT calculation. It is noted that $\alpha_{\text {opt }}$ is approximately proportional to the inverse of the dielectric constant $\varepsilon$ of the material ${ }^{26}$. Since SiC and $\mathrm{Si}$ have $\varepsilon(\mathrm{SiC}) \sim 6.5$ and $\varepsilon(\mathrm{Si}) \sim 11.7$ (ref. 15), respectively, $\alpha_{\text {opt }}$ for $\mathrm{SiC}$ are expected to be increased by a factor of $\sim 2$, compared to that for $\mathrm{Si}$. In our previous hybrid DFT calculation for the $\mathrm{Sn} / \mathrm{Si}(111)$ system (ref. 14), $\alpha=0.2$ was employed to reproduce well the experimental band gap. Thus, $\alpha_{\text {opt }}=0.5$ in the present $\mathrm{Sn} / \mathrm{SiC}(0001)$ system is likely to be reasonable. The $\mathrm{SiC}(0001)$ substrate [with the optimized lattice constant $a_{0}=3.063$ (3.097) $\AA$ for the LDA (GGA) calculation] was modeled by a periodic slab geometry consisting of the eight-layer slab with $\sim 20 \AA$ of vacuum in between the slabs. For the hybrid DFT calculation, we used the lattice constant optimized by the GGA calculation. Each $\mathrm{C}$ atom in the bottom layer of the slab was passivated by one $\mathrm{H}$ atom. The $k$-space integrations for the nonmagnetic (or FM) and AFM structures were done with the $\Gamma$-centered $18 \times 18$ and $9 \times 18$ uniform meshes in the surface Brillouin zones of the $\sqrt{3} \times \sqrt{3}$ and $2 \sqrt{3} \times \sqrt{3}$ unit cells, respectively. All atoms except the bottom $\mathrm{Si}$ and $\mathrm{C}$ layers were allowed to relax along the calculated forces until all the residual force components were less than $0.01 \mathrm{eV} / \AA$.

Dynamical Mean Field Theory calculations. The LDA + DMFT calculation for the single-band Hubbard model in combination with continuous-time quantum Monte Carlo approach as an impurity solver ${ }^{27,28}$. The low-energy effective single-band Hubbard model with on-site Coulomb repulsion $U$ is given by

$$
H=\sum_{\mathrm{k}, \sigma} \varepsilon_{\mathrm{k}, \sigma}^{\mathrm{LDA}}+\sum_{j} U n_{j, \uparrow} n_{j, \downarrow}
$$


where $\varepsilon_{\mathrm{k}, \sigma}^{\mathrm{LDA}}$ are the LDA eigenvalue of the $S_{1}$ state and $n_{j, \sigma}$ is the number operator counting electrons at the Sn-atom site $j$. The DMFT self-consistency equation reads

$$
G_{j, \sigma}\left(i \omega_{n}\right)=\int d \varepsilon_{k} \frac{1}{\left(\begin{array}{cc}
i \omega_{n}+\mu-\sum_{A, \sigma}\left(i \omega_{n}\right) & \varepsilon_{k} \\
\varepsilon_{k} & i \omega_{n}+\mu-\sum_{B, \sigma}\left(i \omega_{n}\right)
\end{array}\right)}
$$

where $\mu$ is the chemical potential, $\omega_{n}$ is the Matsubara frequency, and $j=A, B$. Here, we employed the continuous-time quantum Monte Carlo approach as an impurity solver ${ }^{28}$.

\section{References}

1. Imada, M., Fujimori, A. \& Tokura, Y. Metal-Insulator Transition. Rev. Mod. Phys. 70, 1039 (1998).

2. Meng, Z. Y., Lang, T. C., Wessel, S., Assaad, F. F. \& Muramatsu, A. Quantum Spin Liquid Emerging in Two-dimensional Correlation Dirac Fermions. Nature 464, 847-852 (2010).

3. Northrup, J. E. \& Neugebauer, J. Possibility of a Mott-Hubbard ground state for the SiC(0001) surface. Phys. Rev. B 57, R4230 (1998).

4. Anisimov, V. I., Bedin, A. E., Korotin, M. A., Santoro, G., Scandolo, S. \& Tosatti, E. SiC(0001): A surface Mott-Hubbard insulator. Phys. Rev. B 61, 1752 (2000).

5. Cortes, R. et al. Observation of a Mott Insulating Ground State for Sn/Ge(111) at Low Temperature. Phys. Rev. Lett. 96, 126103 (2006).

6. Modesti, S. et al. Insulating Ground State of $\mathrm{Sn} / \mathrm{Si}(111)-(\sqrt{3} \times \sqrt{3})$ R30 . Phys. Rev. Lett. 98, 126401 (2007).

7. Profeta, G. \& Tosatti, E. Triangular Mott-Hubbard Insulator Phases of Sn/Si(111) and Sn/Ge(111) Surfaces. Phys. Rev. Lett. 98, 086401 (2007).

8. Li, G., Laubach, M., Fleszar, A. \& Hanke, W. Geometrical frustration and the competing phases of the $\mathrm{Sn} / \mathrm{Si}(111) \sqrt{3} \times \sqrt{3} R 30^{\circ}$ surface systems. Phys. Rev. B 83, R041104 (2011).

9. Li, G. et al. Magnetic order in a frustrated two-dimensional atom lattice at a semiconductor surface. Nat. Commun. 4, 1620-1625 (2013).

10. Morikawa, H., Matsuda, I. \& Hasegawa, S. STM observation of Si(111)- $\alpha-\sqrt{3} \times \sqrt{3}$-Sn at low temperature. Phys. Rev. B 65, 2013081 (2002)

11. Carpinelli, J. M., Weitering, H. H., Bartkowiak, M., Stumpf, R. \& Plummer, E. W. Surface Charge Ordering Transition: $\alpha$ Phase of Sn/Ge(111). Phys. Rev. Lett. 79, 2859 (1997).

12. Ballabio, G., Scandolo, S. \& Tosatti, E. $\sqrt{3} \times \sqrt{3} R 30^{\circ}$ versus adatom-rest-atom phases on (111) semiconductor surfaces. Phys. Rev. B 61, R13345 (2000).

13. Lee, J.-H., Kim, H.-J. \& Cho, J.-H. Ferrimagnetic Slater Insulator Phase of the Sn/Ge(111) Surface. Phys. Rev. Lett. 111, 106403 (2013).

14. Lee, J.-H., Ren, X.-Y., Jia, Y. \& Cho, J.-H. Antiferromagnetic superexchange mediated by a resonant surface state in Sn/Si(111). Phys. Rev. B 90, 125439 (2014).

15. Glass, S. et al. Triangular Spin-Orbit-Coupled Lattice with Strong Coulomb Correlations: Sn Atoms on a SiC(0001) Substrate. Phys. Rev. Lett. 114, 247602 (2015).

16. Sato, K. et al. First-principles theory of dilute magnetic semiconductors. Rev. Mod. Phys 82, 1633 (2010).

17. Goodenough, J. B. Theory of the Role of Covalence in the Perovskite-Type Manganites [La, M(II)] MnO . Phys. Rev. 100, 564 (1955).

18. Kanamori, J. Superexchange interaction and symmetry properties of electron orbitals. J. Phys. Chem. Solids 10, 87-98 (1959).

19. Hansmann, P. et al. Long-Range Coulomb Interactions in Surface Systems: A First-Principles Description within Self-Consistently Combined GW and Dynamical Mean-Field Theory. Phys. Rev. Lett. 110, 166401 (2013).

20. Kresse, G. \& Hafner, J. Ab initio molecular dynamics for open-shell transition metals. Phys. Rev. B 48, 13115 (1993).

21. Kresse, G. \& Furthmüller, J. Efficiency of ab-initio total energy calculations for metals and semiconductors using a plane-wave basis set. Comput. Mater. Sci. 6, 15 (1996).

22. Ceperley, D. M. \& Alder, B. J. Ground State of the Electron Gas by a Stochastic Method. Phys. Rev. Lett. 45, 566 (1980).

23. Perdew, J. P., Burke, K. \& Ernzerhof, M. Generalized Gradient Approximation Made Simple. Phys. Rev. Lett. 77, 3865 (1996).

24. Heyd, J., Scuseria, G. E. \& Ernzerhof, M. Hybrid functionals based on a screened Coulomb potential. J. Chem. Phys. 118, 8207 (2003).

25. Krukau, A. V., Vydrov, O. A., Izmaylov, A. F. \& Scuseria, G. E. Influence of the exchange screening parameter on the performance of screened hybrid functionals. J. Chem. Phys. 125, 224106 (2006)

26. Marques, M. A. L., Vidal, J., Oliveira, M. J. T., Reining, L. \& Botti, S. Density-based mixing parameter for hybrid functionals. Phys. Rev. B 83, 035119 (2011).

27. Georges, A., Kotliar, G., Krauth, W. \& Rozenberg, M. J. Dynamical mean-field theory of strongly correlated fermion systems and the limit of infinite dimensions. Rev. Mod. Phys. 68, 13 (1996).

28. Gull, E., Millis, A. J., Lichtenstein, A. I., Rubtsov, A. N., Troyer, M. \& Werner, P. Continuous-time Monte Carlo methods for quantum impurity models. Rev. Mod. Phys. 83, 349 (2011).

\section{Acknowledgements}

This work was supported by National Research Foundation of Korea (NRF) grant funded by the Korea Government (MSIP) (2015R1A2A2A01003248). The calculations were performed by KISTI supercomputing center through the strategic support program (KSC-2015-C3-017) for the supercomputing application research.

\section{Author Contributions}

S.Y. and J.-H. Choi performed the DFT calculations. H.L. performed the LDA + DMFT calculations. J.-H. Cho designed the study and wrote the paper. All authors interpreted the results and reviewed the manuscript.

\section{Additional Information}

Supplementary information accompanies this paper at http://www.nature.com/srep

Competing financial interests: The authors declare no competing financial interests.

How to cite this article: Yi, S. et al. Nature of the Insulating Ground State of the Two-Dimensional Sn Atom Lattice on $\mathrm{SiC}(0001)$. Sci. Rep. 6, 30598; doi: 10.1038/srep30598 (2016). 
(c) (i) This work is licensed under a Creative Commons Attribution 4.0 International License. The images or other third party material in this article are included in the article's Creative Commons license, unless indicated otherwise in the credit line; if the material is not included under the Creative Commons license, users will need to obtain permission from the license holder to reproduce the material. To view a copy of this license, visit http://creativecommons.org/licenses/by/4.0/

(C) The Author(s) 2016 